\title{
SNOW PHENOMENA MODELING THROUGH ONLINE PUBLIC MEDIA
}

\author{
Roman Fedorov, Piero Fraternali, Marco Tagliasacchi \\ Politecnico di Milano \\ Dipartimento di Elettronica, Informazione e Bioingegneria \\ Piazza Leonardo da Vinci, 32 - 20133 Milan - Italy
}

\begin{abstract}
We propose a method for the environmental monitoring through the publicly available media User Generated Content (UGC). In particular we address the problem of the snow cover and level estimation by analyzing the social media data such as geo-tagged photographs and public webcams installed in mountain regions. The entire pipeline of the process is presented to the audience: from the data crawling and automatic relevance classification (does or does not the photograph contain a significant mountain profile) to the image content analysis and environmental models (identification of the snow covered area on the photograph). Each presented component is self-contained and can be inspected individually, the connections between the components however are strongly highlighted allowing the viewer to understand intuitively the entire pipeline structure.
\end{abstract}

Index Terms - UGC, environmental modeling, virtual sensors, object identification, scene classification

\section{INTRODUCTION AND MOTIVATIONS}

The amount of media User Generated Content (UGC) on the Web is growing exponentially nowadays [1], and so is also doing the amount of the latent implicit knowledge contained within it. We studied the problem of this knowledge retrieval in a field lacking well spatially and temporary sampled data - environmental monitoring. In particular, the problem of the Italian Alps snow cover and level map estimation is currently addressed by the Italian Regional Environmental Protection Agencies by interpolating the data gathered from physical snow measuring stations, spatially density of which is totally insufficient for the generation of a precise snow map.

The aim of the project is to explore the potential contribution of the online media UGC to the precision of existing environmental phenomena models. We consider each geo-tagged photograph with a meaningful mountain profile as a source of a single virtual snow measure. Each webcam framing a

This work is supported by the EU FP7 CUbRIK Integrating Project, http://www.cubrikproject.eu and POR-FESR 2007-2013 PROACTIVE Project, http://www.proactiveproject.eu mountain instead is seen as an entire virtual snow measuring station. This work involves several number of challenging tasks such as the identification of the mountains present on the images and detecting the snow covered area on that mountains.

\section{SCIENTIFIC AND TECHNICAL DESCRIPTION}

The goal of the proposed method is to autonomously collect input visual data, process it and produce a snow measurement model for a certain geographical area. The entire procedure can be split in four different independent steps (represented schematically in Fig. 1), in fact a separate component for each step was developed, and is individually discussed.

\subsection{Relevant photograph crawling}

The proposed system has two main sources of input data: geo-tagged photographs that contain a meaningful mountain profile and webcams framing mountain regions. The purpose of the first component is so to retrieve the largest possible amount of mountain photographs in a given geographical area.

Given the source of the photographs (photo hosting platform, social network) all the images available in the defined area along with their geo-tags are retrieved. The fraction of the photographs relevant to our purpose however is low enough to generate a high rate of false positives even with a highly accurate classification algorithm. For this reason a filtering based on the altitude of the photographer during the shot is applied: having the geographical position of the shot and the Digital Elevation Model (DEM) of that area it comes simple to estimate the altitude of the photographer, so all the photographs taken under a certain altitude threshold are discarded. The intuition behind this thresholding is the fact that a picture taken in the mountain has a high probability to frame another mountain. A supervised learning Support Vector Machine (SVM) then performs a two-class classification, splitting the collection in photographs that contain clear mountain profiles (relevant photographs) and those that do not. All photographs considered relevant are then passed to the next component. 


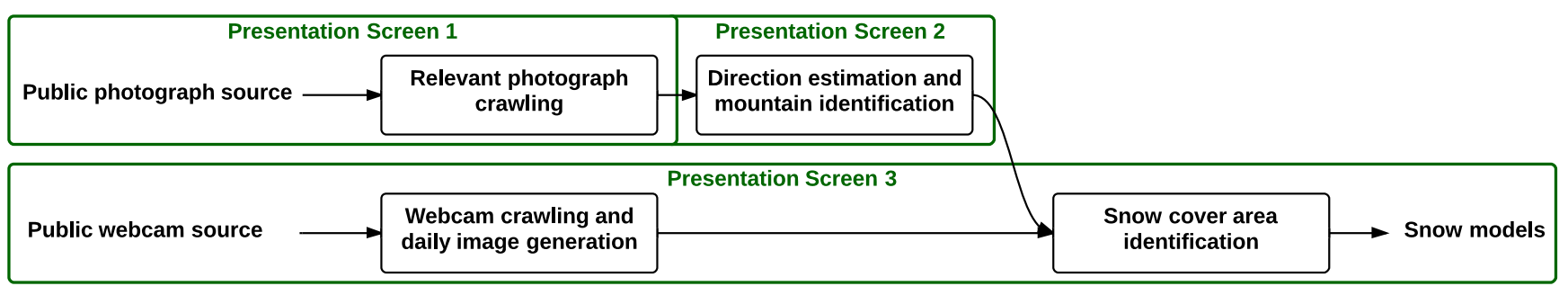

Fig. 1. The proposed pipeline schema with its components and the corresponding presentations.

\subsection{Photograph mountain identification}

Given as input a geo-tagged photograph, the component estimates its Field Of View (FOV) and the direction of the camera using a matching algorithm on the photograph edge maps and a rendered view of the mountain silhouettes that should be seen from the observer's point of view. This view is generated from the DEM of the corresponding geographical area [2]. The refinement step then identifies the mountain peaks present in the photograph and their profiles. Once the direction of the photograph is known the snow coverage estimation component is invoked.

\subsection{Webcam crawling and daily image generation}

A webcam placed in a mountain region can be seen as a source of relevant photographs with a high temporal resolution (usually in the range $1^{\prime}-15^{\prime}$ ). Cloudy meteorological conditions however are very common to be found at high altitudes, so a significant portion of the produced images ( $60 \%$ in the monitored sample) is compromised by the clouds covering partially or totally the mountains. This problem is explicitly addressed by the component that considers instead a webcam as a source of daily photographs, each pixel of which is defined as the median value between the corresponding pixels of all the images produced by the webcam during that day. The assumption underlying this idea is the fact that although the clouds are often present on the webcam images, the area covered by the clouds is usually reduced and is constantly moving due to the wind. This assumption turned out to be true in all the monitored cases except those where the meteorological conditions were so adverse as not to show the mountain panorama at any time of the day. The daily photograph in these cases is usually made of a constant cloud/fog background only, and is discarded by an algorithm based on the color statistics of the image.

\subsection{Snow cover area identification}

Once the direction of the photograph is estimated the portion of the photograph representing the mountains can be extracted by considering only the pixels of the rendered DEM view that correspond to the terrain points at an altitude above a defined threshold. In case of a webcam (given the static nature of the frame) the mountain area is defined by a mask generated manually for each webcam. All the pixels belonging to the mountain area are then classified as snow/non snow pixels by a statistical analysis of the blue component of the image as proposed by Salvatori et al. [3].

\section{IMPLEMENTATION AND USE}

We designed our contribution to this session presenting each of the components described in the Section 2 with an interactive an appealing approach. Although each component is selfcontained and can be inspected individually, the connections between the components are strongly highlighted allowing the viewer to understand intuitively the entire pipeline structure. The entire proposal includes three presentation screens placed one next to each other, that follow faithfully the schema described in Fig. 1. The different graphical user interfaces are briefly shown in Fig. 2, instead the videos showing faithfully the interface, functioning and the possible audience interaction with each of the presentation screens are available at the project website. http://bit.ly/icip2014demo

A default set of representative mountain photographs will be provided to the viewers that want to try the components themselves, though a viewer will be able and will be encouraged to use any photograph of his ownership. An own photograph can be uploaded connecting any external storage device or simply sending the image to a specific email address that will be created.

\subsection{Presentation screen 1}

Our study concerns a $300 \times 160 \mathrm{~km}$ region across the Italian and Swiss Alps: Flickr contains a total number of 600k photographs with a valid geo-tag within this area. A crowdsourcing experiment on a random sample of these images showed that the $21 \%$ of the photographs taken above $600 \mathrm{~m}$ of altitude contain a distinctive skyline of the mountain profile. The photographs taken above this threshold are so used for further analysis.

An interface implementing a heat map as a function of the number of crawled photographs in the defined geographical area can be explored by the viewer: defining a custom subarea a detailed statistics on the number of photographs 


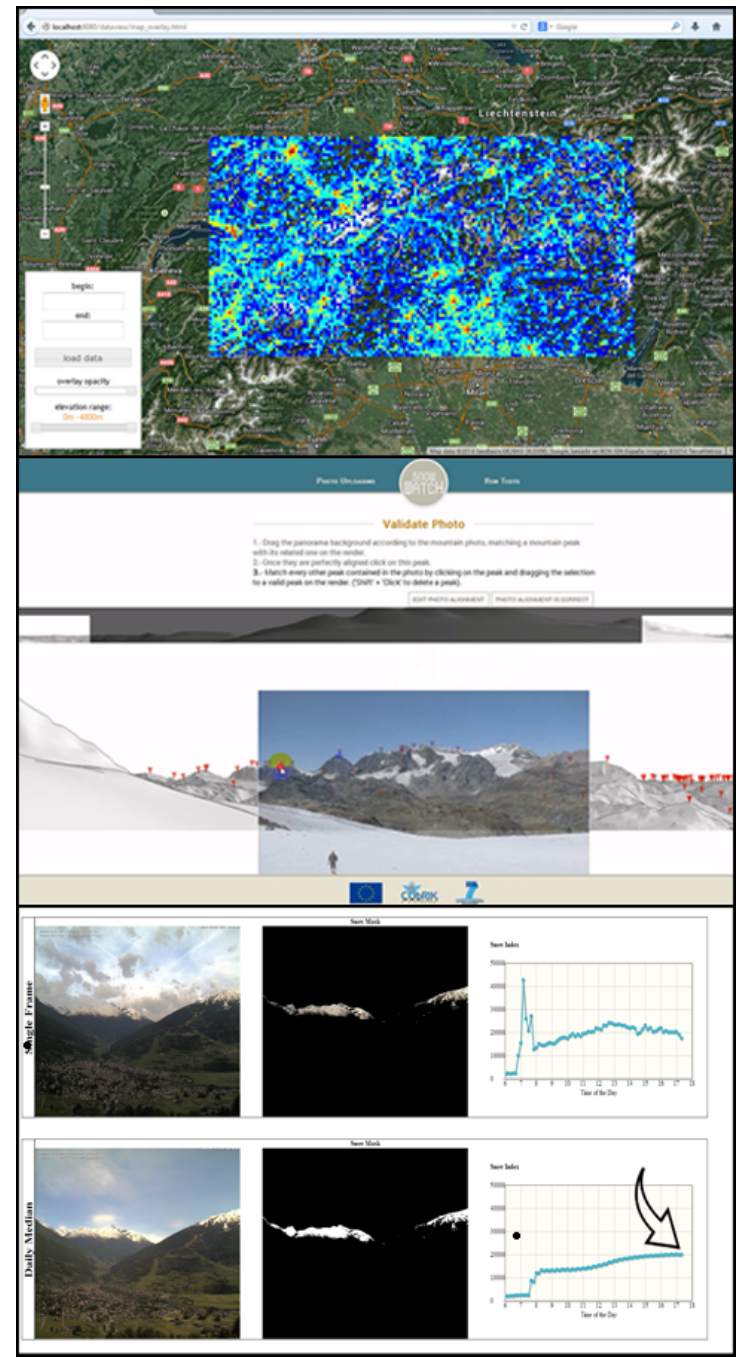

Fig. 2. Graphical user interface of first (top), second (middle) and third (bottom) presentation screen.

through time is displayed along with a sample of the photographs classified as containing mountains. The viewer can navigate freely the heat map combined with a satellite view, exploring the photograph density distribution with respect to the Alpine mountain positions or alternatively just upload any photograph of his choice testing the classifier ability to discard non mountain images.

\subsection{Presentation screen 2}

Once the viewer chose a geo-tagged photograph containing a mountain profile to be detected, the DEM rendered view is generated and displayed. The tool then displays its guess on the best overlapping position between the photograph and the rendered view. If the viewer considers it to be wrong, the position can be manually corrected, allowing eventually the viewer to realize that the problem was set up incorrectly due to the absence of any possible overlap (this situation may arise when the geo-tag of the photograph is imprecise). Once the overlapping correctness is confirmed or the correction is manually applied the input photograph enriched with the mountain peak names and information is generated and presented as the final result.

\subsection{Presentation screen 3}

Since the algorithm is based on the whole daily history of a given webcam, it is not possible to apply the procedure to any webcam chosen by the viewer on the fly. For this reason this part of the presentation is forced to be less interactive with respect to others but still the most visually attractive. When an item from the list of pre-cralwed webcams is chosen, the median photograph iterative approach is shown (scrolling through the daily pictures with moving clouds a summarized and clouds free daily photograph is built), then the snow pixel classifier displays the portion of the photograph considered snow along with an estimated modeled snow measure. The whole animation is cycled day by day giving the audience an intuitive idea of the snow level change in time.

\section{CONCLUSIONS AND FUTURE DEVELOPMENTS}

We presented a tool for the extraction of the environmental data from media content and its aggregation. An interactive presentation program divided in three components was developed for this session. The novelty of the approach resides in the use of the highly available public UGC and a method that is robust to the noise of the uncontrolled input data, such as bad image pose, bad weather conditions, wrong time stamp, imprecise geo-tag.

Future developments of this project will include the study of the connections between the snow models produced and the real environmental phenomena trends: snow cover, snow level, daily precipitations, temperature.

\section{REFERENCES}

[1] Aiden R. Doherty and Alan F. Smeaton, "Automatically augmenting lifelog events using pervasively generated content from millions of people," Sensors, vol. 10, no. 3, pp. 1423-1446, 2010.

[2] Roman Fedorov, Davide Martinenghi, Marco Tagliasacchi, and Andrea Castelletti, "Exploiting user generated content for mountain peak detection," .

[3] Rosamaria Salvatori, Paolo Plini, Marco Giusto, Mauro Valt, Roberto Salzano, Mauro Montagnoli, Anselmo Cagnati, Giuseppe Crepaz, and Daniele Sigismondi, "Snow cover monitoring with images from digital camera systems," Ital. J. Remote Sens, vol. 43, pp. 137-145, 2011. 